\title{
Finite Elements Approaches in the Solution of Field Functions in Multidimensional Space: A Case of Boundary Value Problems
}

\section{Chukwutoo Christopher Ihueze* ${ }^{1}$, Okafor Emeka Christian ${ }^{1}$, Edelugo Sylvester Onyemaechi $^{2}$}

\author{
${ }^{1}$ Department of Industrial/Production Engineering, Nnamdi Azikiwe University Awka. \\ ${ }^{2}$ Department of Mechanical Engineering University of Nigeria \\ *Corresponding Author: ihuezechukwutoo@yahoo.com
}

\begin{abstract}
An idealized two dimensional continuum region of GRP composite was used to develop an efficient method for solving continuum problems formulated for space domains. The continuum problem is solved by minimization of a functional formulated through a finite element procedure employing triangular elements and assumption of linear approximation polynomial. The assemblage of elements functional derivatives system of equations through FEM assembly procedure made possible the definition of a unique and parametrically defined model from which the solution of continuum configuration with an arbitrary number of scales is solved. The finite element method(FEM )developed is recommended to be applied in the evaluation of the function of functions in irregular shaped continuum whose boundary conditions are specified such as in the evaluation of displacement in structures and solid mechanics problems, evaluation of temperature distribution in heat conduction problems, evaluation of displacement potential in acoustic fluids evaluation of pressure in potential flows, evaluation of velocity in general flows, evaluation of electric potential in electrostatics, evaluation of magnetic potential in magnetostatics and in the solution of time dependent field problems. A unified computational model with standard error of 0.15 and correlation coefficient of 0.72 was developed to aid analysis and easy prediction of regional function with which the continuum function was successfully modeled and optimized through gradient search and Lagrange multipliers approach. Above all the optimization schemes of gradient search and Lagrangian multiplier confirmed local minimum of function as 0.006-0.00847 to confirm the predictions of FEM and constraint conditions.
\end{abstract}

Keywords: finite element, continuum, functional of function, extremum, boundary value 


\section{INTRODUCTION}

In calculus of variations, instead of attempting to locate points that extremize function of one or more variables that extremize quantities called functional, functions of functions that extremize the functional are found [1]. Also in the finite element process an approximate solution is sought to the problem of minimizing a functional. The concept of the finite element approach to elasticity as a process in which the total potential energy is minimized with respect to nodal displacements can obviously be extended to a variety of physical problems in which an extremum principle exists. The two concepts are combined in this study. Zienkiewicz and Cheung [2] applied similar approach to solve continuum problem expressed in derivative format employing the concept of functional minimization with FEM.

Above all, there are many problems encountered in engineering and physics where the minimization of the integrated quantity usually referred as functional and subject to some boundary conditions results in the exact solution. This functional may represent a physical recognizable variable in some instances, for many purposes it is simply a mathematically defined entity.

The geometry of field quantities or continuum may be a problem to close form solution of field functions encountered in engineering and science that appropriate algorithm becomes necessary to obtain optimum solution, it is then necessary to employ calculus of variation principles and FEM to obtain optimum continuum field functions whose boundary conditions are specified. The engineering field continuum problems can be basically in form of wave phenomenon, diffusion phenomenon and potential phenomenon usually represented by hyperbolic, parabolic and elliptic differential equations respectively [3].

The objective of this study is therefore to present a methodical approach to solve multiple dimensional field problems using integrated variational and FEM approach to establish relations for all elements functional of continuum where the minimization of the elements functionals system and solution are expected to give the stationary values of the function which extremize the functional.

\section{THEORETICAL BACKGROUND}

A finite element model of a two dimensional quadratic function is expected to present a methodical approach to employ for solution of multidimensional field functions that may have regular or irregular field regions. Zienkiewicz and Cheung [2] presented Euler theorem to approximate field functions if the integral or functional of the form

$$
I(u)=\iiint f\left(x, y, z, u, \frac{\partial u}{\partial x}, \frac{\partial u}{\partial y}, \frac{\partial u}{\partial z}\right) d x d y d z
$$


is to be minimized. The necessary and sufficient condition for this minimum to be reached is that the unknown function $\mathrm{u}(\mathrm{x}, \mathrm{y}, \mathrm{z})$ should satisfy the following differential equation

$$
\frac{\partial}{\partial x}\left[\frac{\partial f}{\partial(\partial u / \partial x)}\right]+\frac{\partial}{\partial y}\left[\frac{\partial f}{\partial(\partial u / \partial y)}\right]+\frac{\partial}{\partial z}\left[\frac{\partial f}{\partial(\partial u / \partial z)}\right]-\frac{\partial f}{\partial u}=0
$$

within the same region, provided u satisfies the same boundary conditions in both cases, while the equation governing the behaviour of unknown physical quantity u can generally be expressed as

$$
\frac{\partial}{\partial x}\left(k_{x} \frac{\partial u}{\partial x}\right)+\frac{\partial}{\partial y}\left(k_{y} \frac{\partial u}{\partial y}\right)+\frac{\partial}{\partial z}\left(k_{z} \frac{\partial u}{\partial z}\right)+Q=0
$$

where

$\mathrm{u} \quad=$ unknown function assumed to be single valued within the region

$\mathrm{k}_{\mathrm{x}}, \mathrm{k}_{\mathrm{y}}, \mathrm{k}_{\mathrm{z}}, \mathrm{Q}=$ specified functions of $\mathrm{x}, \mathrm{y}, \mathrm{z}$

$\mathrm{x}, \mathrm{y}, \mathrm{z}=$ space variables

The equivalent formulation to that of equation (3) is the requirement that the volume integral given below and taken over the whole region, should be

$\chi=\iiint\left\{\frac{1}{2}\left[\mathrm{k}_{\mathrm{x}}\left(\frac{\partial \mathrm{u}}{\partial \mathrm{x}}\right)^{2}+\mathrm{k}_{\mathrm{y}}\left(\frac{\partial \mathrm{u}}{\partial \mathrm{y}}\right)^{2}+\mathrm{k}_{\mathrm{z}}\left(\frac{\partial \mathrm{u}}{\partial \mathrm{z}}\right)^{2}\right]-\mathrm{Q} \mathrm{u}\right\} \mathrm{dxdydz}$

subject to $\mathrm{u}$ obeying the same boundary conditions.

For two dimensional differential equation representing some physical quantities then

$\chi=\iint\left\{\frac{1}{2}\left[\mathrm{k}_{\mathrm{x}}\left(\frac{\partial \mathrm{u}}{\partial \mathrm{x}}\right)^{2}+\mathrm{k}_{\mathrm{y}}\left(\frac{\partial \mathrm{u}}{\partial \mathrm{y}}\right)^{2}\right]-\mathrm{Q} \mathrm{u}\right\} \mathrm{dxdy}$

For the case of our interest, the equivalent functional to be minimized for 2-D Laplace model reduces to

$\chi=\iint\left\{\frac{1}{2}\left[\mathrm{k}_{\mathrm{x}}\left(\frac{\partial \mathrm{u}}{\partial \mathrm{x}}\right)^{2}+\mathrm{k}_{\mathrm{y}}\left(\frac{\partial \mathrm{u}}{\partial \mathrm{y}}\right)^{2}\right]\right\} \mathrm{dxdy}$

The finite element version of an integrated functional is obtained and minimized with respect to degrees of freedoms of the associated elements. The element functional equations are assembled and boundary conditions applied, resulting in a system of equations equal to the number of unconstrained degrees of freedoms of the continuum. 


\section{FINITE ELEMENT METHOD (FEM)}

Euler variational minimum integral theorem was applied with the procedure of [4] on the general equation governing the behavior of field functions presented by [2] to develop a finite element version of elements functions functionals. The elements function functionals are minimized with respect to degrees of freedoms in the finite element method of assembly are applied to obtain the system model that is solved for the field of function . Basic approaches to achieve finite element solouttion of continuum are also available in [5-8\}.

\subsection{Formulation of Finite Elements Equations}

The elements functional of the study are derived for each element and minimized using equation (6). Minimization of element functional entails finding the partial derivatives of the element functional at its nodes. The contributions of each element nodes are established and added for all continuum nodes to obtain the finite element model of the system. The formulation of finite element model starts by choosing the element type and then choosing the approximation polynomial coefficients are determined for establishing the element equations from where the interpolation functions for $\mathrm{u}$ are established for all elements. This function $\mathrm{u}$ is used then employed in finding the finite element model of the elements functionals from where the sought functions are found.

\subsubsection{Discritization and element topology description}

The region is discretized into 16 triangular elements with 26 degrees of freedom and assuming displacement in the global system of coordinate (horizontal direction only) only as in Figure 1 elements topologies are described in Table 1 for the establishment of element interpolation functions for the functional equations for the finite element minimization scheme.

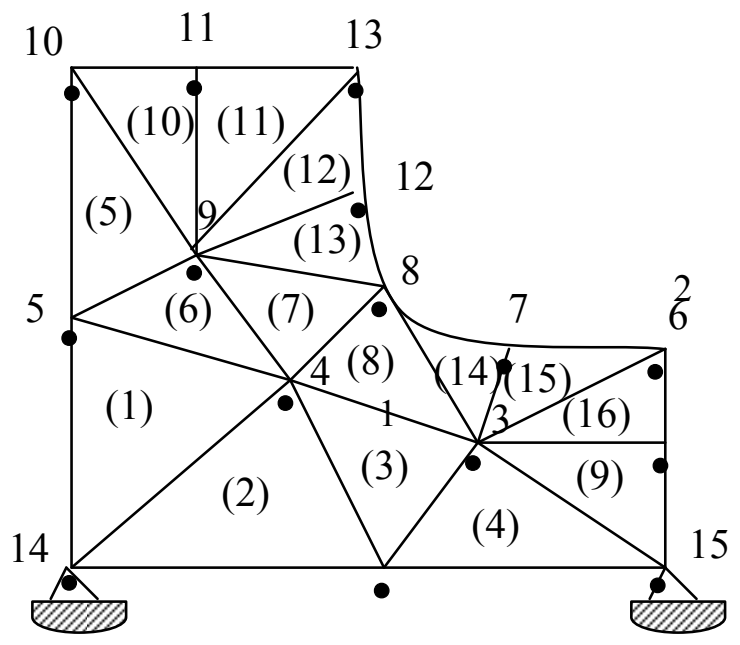

Figure 1: Idealized Finite element Model of two Dimensional Composite Body. 
Table 1: Element Topology Description.

\begin{tabular}{|l|l|l|l|}
\hline $\begin{array}{l}\text { Element } \\
\text { Number }\end{array}$ & $\begin{array}{l}\text { Active degrees of freedom } \\
\text { of elements }\end{array}$ & Element coordinates & $\begin{array}{l}\text { Element } \\
\text { nodes }\end{array}$ \\
\hline 1 & $\mathrm{u}_{5}, \mathrm{u}_{4}, \mathrm{u}_{14}, \mathrm{v}_{5}, \mathrm{v}_{4}, \mathrm{v}_{14}$ & $(0,0),(0,21),(16,16)$ & $14,5,4$ \\
\hline 2 & $\mathrm{u}_{1}, \mathrm{u}_{4}, \mathrm{u}_{14}, \mathrm{v}_{1}, \mathrm{v}_{4}, \mathrm{v}_{14}$ & $(0,0),(16,16),(21,0)$ & $14,4,1$ \\
\hline 3 & $\mathrm{u}_{1}, \mathrm{u}_{4}, \mathrm{u}_{3}, \mathrm{v}_{1}, \mathrm{v}_{4}, \mathrm{v}_{3}$ & $(21,0),(16,16),(25,10)$ & $1,4,3$ \\
\hline 4 & $\mathrm{u}_{1}, \mathrm{u}_{3}, \mathrm{u}_{15}, \mathrm{v}_{1}, \mathrm{v}_{3}, \mathrm{v}_{15}$ & $(21,0),(21,0),(25,10)$ & $1,3,15$ \\
\hline 5 & $\mathrm{u}_{5}, \mathrm{u}_{10}, \mathrm{u}_{9}, \mathrm{v}_{5}, \mathrm{v}_{10}, \mathrm{v}_{9}$ & $(0,21),(0,37),(10,25)$ & $5,10,9$ \\
\hline 6 & $\mathrm{u}_{5}, \mathrm{u}_{9}, \mathrm{u}_{4}, \mathrm{v}_{5}, \mathrm{v}_{9}, \mathrm{v}_{4}$ & $(0,21),(0,25),(16,16)$ & $5,9,4$ \\
\hline 7 & $\mathrm{u}_{9}, \mathrm{u}_{4}, \mathrm{u}_{8}, \mathrm{v}_{9}, \mathrm{v}_{4}, \mathrm{v}_{8}$ & $(16,16),(10,25),(22,13)$ & $4,9,8$ \\
\hline 8 & $\mathrm{u}_{8}, \mathrm{u}_{4}, \mathrm{u}_{3}, \mathrm{v}_{8}, \mathrm{v}_{4}, \mathrm{v}_{3}$ & $(16,16),(22,23),(25,10)$ & $4,8,3$ \\
\hline 9 & $\mathrm{u}_{15}, \mathrm{u}_{3}, \mathrm{u}_{2}, \mathrm{v}_{15}, \mathrm{v}_{3}, \mathrm{v}_{2}$ & $(25,10),(35,10),(35,0)$ & $15,3,2$ \\
\hline 10 & $\mathrm{u}_{9}, \mathrm{u}_{10}, \mathrm{u}_{11}, \mathrm{v}_{9}, \mathrm{v}_{10}, \mathrm{v}_{11}$ & $(0,37),(10,37),(10,25)$ & $9,10,11$ \\
\hline 11 & $\mathrm{u}_{9}, \mathrm{u}_{11}, \mathrm{u}_{13}, \mathrm{v}_{9}, \mathrm{v}_{11}, \mathrm{v}_{13}$ & $(10,25),(10,37),(18,37)$ & $9,11,13$ \\
\hline 12 & $\mathrm{u}_{9}, \mathrm{u}_{13}, \mathrm{u}_{12}, \mathrm{v}_{9}, \mathrm{v}_{13}, \mathrm{v}_{12}$ & $(10,25),(18,37),(19,29)$ & $9,13,12$ \\
\hline 13 & $\mathrm{u}_{9}, \mathrm{u}_{12}, \mathrm{u}_{8}, \mathrm{v}_{9}, \mathrm{v}_{12}, \mathrm{v}_{8}$ & $(10,25),(19,29),(22,23)$ & $9,12,8$ \\
\hline 14 & $\mathrm{u}_{3}, \mathrm{u}_{8}, \mathrm{u}_{7}, \mathrm{v}_{3}, \mathrm{v}_{8}, \mathrm{v}_{7}$ & $(25,10),(22,23),(29,19)$ & $3,8,7$ \\
\hline 15 & $\mathrm{u}_{3}, \mathrm{u}_{7}, \mathrm{u}_{6}, \mathrm{v}_{3}, \mathrm{v}_{7}, \mathrm{v}_{6}$ & $(25,11),(29,19),(35,18)$ & $3,7,6$ \\
\hline 16 & $\mathrm{u}_{3}, \mathrm{u}_{6}, \mathrm{u}_{12}, \mathrm{v}_{3}, \mathrm{v}_{6}, \mathrm{v}_{12}$ & $(25,10),(35,18),(35,10)$ & $3,6,2$ \\
\hline & & & \\
\hline
\end{tabular}

\subsection{Determination of FEM Characteristics}

\subsubsection{Element 1 interpolation and functional equation formulation}
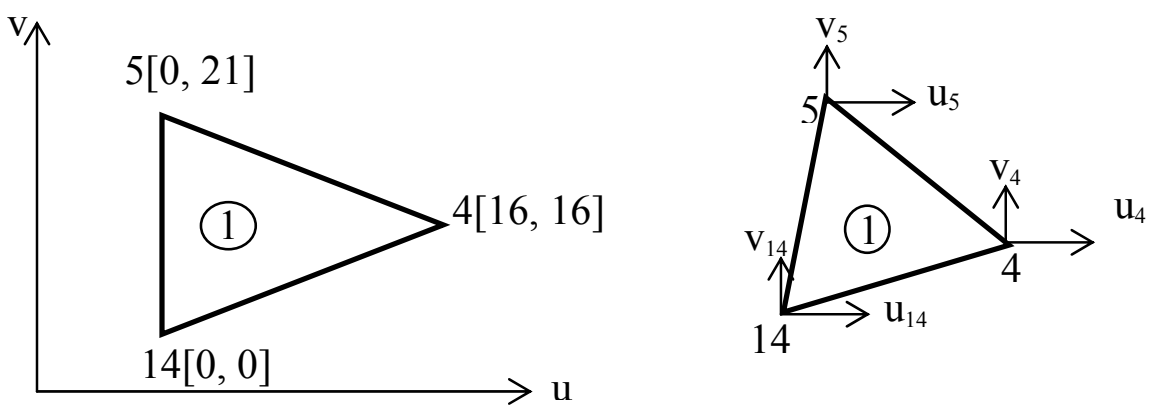

By assuming a linear approximation polynomial of the form

$$
u(x, y)=a_{0}+a_{1} x+a_{2} y
$$

and following the method of Ihueze etal (2009) and Asterly (1992) 
Where $\mathrm{a}_{0}, \mathrm{a}_{1}, \mathrm{a}_{2}$ are called polynomial coefficients or shape constants so that by passing (1) through the nodes of element 1the system of unknown function of the element becomes:

$$
\begin{aligned}
& u_{14}=a_{0}+a_{1} x_{14}+a_{2} y_{14} \\
& u_{4}=a_{0}+a_{1} x_{4}+a_{2} y_{4} \\
& u_{5}=a_{0}+a_{1} x_{5}+a_{2} y_{5}
\end{aligned}
$$

Putting the above polynomial function in matrix form then

$$
\left[\begin{array}{lll}
1 & x_{14} & y_{14} \\
1 & x_{4} & y_{4} \\
1 & x_{5} & y_{5}
\end{array}\right]\left[\begin{array}{l}
a_{0} \\
a_{1} \\
a_{2}
\end{array}\right]=\left[\begin{array}{ll}
u_{14} \\
u_{4} \\
u_{5}
\end{array}\right]
$$

By applying Crammers rule

$$
\begin{aligned}
& a_{0}=\frac{1}{2 A}\left\{u_{14}\left(x_{4} y_{5}-x_{5} y_{4}\right)+u_{4}\left(x_{5} y_{14}-x_{14} y_{5}\right)+u_{5}\left(x_{14} y_{4}-x_{4} y_{14}\right)\right\} \\
& a_{1}=\frac{1}{2 A}\left\{u_{14}\left(y_{4}-y_{5}\right)+u_{4}\left(y_{5}-y_{14}\right)+u_{5}\left(y_{14}-y_{4}\right)\right. \\
& a_{2}=\frac{1}{2 A}\left\{u_{14}\left(x_{5}-x_{4}\right)+u_{4}\left(x_{14}-x_{5}\right)+u_{5}\left(x_{4}-x_{14}\right)\right.
\end{aligned}
$$

Substituting (2) in (1) then

$$
\begin{aligned}
u_{1}= & a_{0}+a_{1} x+a_{2} y \\
& u_{1}=\frac{1}{2 A}\left\{u_{14}\left(x_{4} y_{5}-x_{5} y_{4}\right)+u_{4}\left(x_{5} y_{14}-x_{14} y_{5}\right)+u_{5}\left(x_{14} y_{4}-x_{4} y_{14}\right)\right\}+ \\
& \frac{1}{2 A}\left\{u_{14}\left(y_{4}-y_{5}\right)+u_{4}\left(y_{5}-y_{14}\right)+u_{5}\left(y_{14}-y_{4}\right)\right\} x+ \\
& \frac{1}{2 A}\left\{u_{14}\left(x_{5}-x_{4}\right)+u_{4}\left(x_{14}-x_{5}\right)+u_{5}\left(x_{4}-x_{14}\right)\right\} y
\end{aligned}
$$

Recall that the approximation function is given as

$$
u=N_{14} u_{14}+N_{4} u_{4}+N_{5} u_{5}
$$

Comparing (5) and (6) we evaluate shape and interpolation function thus 
$\left.\begin{array}{l}N_{14}(x, y)=\frac{1}{2 A}\left\{\left(x_{4} y_{5}-x_{5} y_{4}\right)+\left(y_{4}-y_{5}\right) x+\left(x_{5}-x_{4}\right)\right\} y \\ N_{4}(x, y)=\frac{1}{2 A}\left\{\left(x_{5} y_{14}-x_{14} y_{5}\right)+\left(y_{5}-y_{14}\right) x+\left(x_{14}-x_{5}\right)\right\} y \\ N_{5}(x, y)=\frac{1}{2 A}\left\{\left(x_{14} y_{4}-x_{4} y_{14}\right)+\left(y_{14}-y_{4}\right) x+\left(x_{4}-x_{14}\right)\right\} y\end{array}\right\}$

But $\mathrm{A}=\frac{1}{2}\left\{\left(x_{4} y_{5}-x_{5} y_{4}\right)+\left(x_{5} y_{14}-x_{14} y_{5}\right)+\left(x_{14} y_{4}-x_{4} y_{14}\right)\right\}=168 \mathrm{~mm}^{2}$

where $\mathrm{A}=$ area of triangular element so that

$$
\begin{aligned}
& N_{14}=\frac{1}{336}(336-5 x-16 y) \\
& N_{4}=\frac{1}{336}(21 x) \\
& N_{5}=\frac{1}{336}(-16 x+16 y)
\end{aligned}
$$

Substituting (7) in (4)

$$
\left.\begin{array}{l}
u=\frac{1}{336}(336-5 x-16 y) u_{14}+\frac{1}{336}(21 x) u_{4}+\frac{1}{336}(-16 x+16 y) u_{5} \\
\frac{\partial u}{\partial x}=\frac{-5 u_{14}}{336}+\frac{21 u_{4}}{336}-\frac{16 u_{5}}{336} \\
\frac{\partial u}{\partial y}=\frac{-16 u_{14}}{336}+\frac{16 u_{5}}{336}
\end{array}\right\}
$$

By assuming a two dimensional Laplace function for the continuum function of the form

$\frac{\partial^{2} u}{\partial x^{2}}+\frac{\partial^{2} u}{\partial y^{2}}=0$

The minimum function integral called functional to be minimized becomes in which case

$\mathrm{kx}=\mathrm{ky}=\mathrm{kz}=1$ and $\mathrm{Q}=0$

So that (4) reduces to

$$
x=\iint\left[\frac{1}{2}\left\{\left(\frac{\partial u}{\partial x}\right)^{2}+\left(\frac{\partial u}{\partial y}\right)^{2}\right\}\right] d x d y
$$


By substitutingg the first partial derivatives of the element 4 interpolation functions in (12) with $d x d y=\mathrm{A}=168$

$$
x=0.418 u_{14}^{2}-0.313 u_{4} u_{14}-0.524 u_{5} u_{14}-u_{4} u_{5}-0.656 u_{4}^{2}+0.762 u_{5}^{2}
$$

By differentiating w.r.t. $\mathrm{u}_{14}, \mathrm{u}_{4}$, and $\mathrm{u}_{5}$

$$
\left.\begin{array}{l}
\frac{\partial x}{\partial u_{14}}=\left(0.836 u_{14}-0.313 u_{4}-0.524 u_{5}\right) * 0.5 \\
\frac{\partial x}{\partial u_{4}}=\left(1.312 u_{4}-0.313 u_{14}-u_{5}\right) * 0.5 \\
\frac{\partial x}{\partial u_{5}}=\left(1.524 u_{5}-0.524 u_{14}-u_{4}\right) * 0.5
\end{array}\right)
$$

\subsubsection{Element 2 interpolation and functional equation formulation}
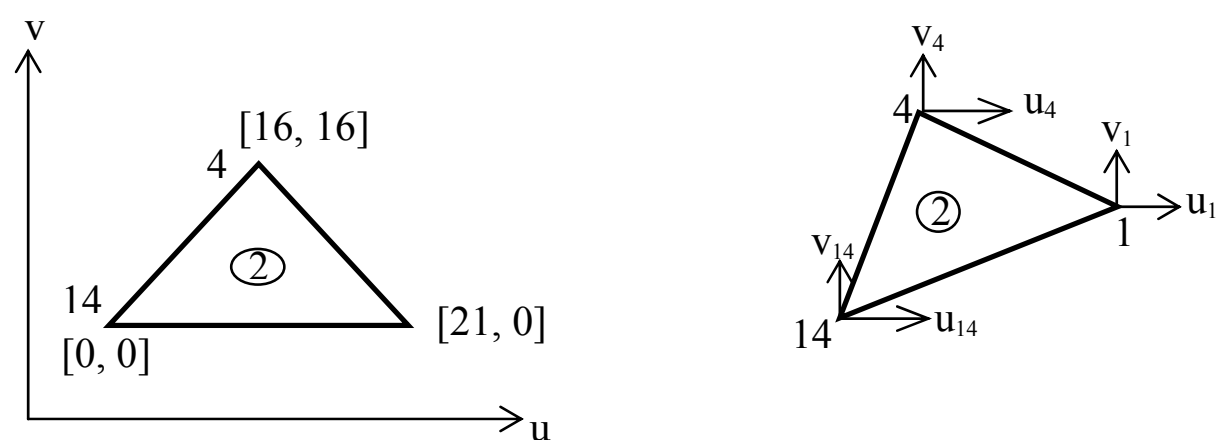

By assuming a linear approximation polynomial of the form

$u(x, y)=a_{0}+a_{1} x+a_{2} y$

Passing (15) through the nodes then

$$
\begin{aligned}
& u_{14}=a_{0}+a_{1} x_{14}+a_{2} y_{14} \\
& u_{1}=a_{0}+a_{1} x_{1}+a_{2} y_{1} \\
& u_{4}=a_{0}+a_{1} x_{4}+a_{2} y_{4}
\end{aligned}
$$

Putting the system in matrix form then

$$
\left[\begin{array}{lll}
1 & x_{14} & y_{14} \\
1 & x_{1} & y_{1} \\
1 & x_{4} & y_{4}
\end{array}\right]\left[\begin{array}{l}
a_{0} \\
a_{1} \\
a_{2}
\end{array}\right]=\left[\begin{array}{l}
u_{14} \\
u_{1} \\
u_{4}
\end{array}\right]
$$


By applying Crammers rule,

$$
\left.\begin{array}{l}
a_{0}=\frac{1}{2 A}\left\{u_{14}\left(x_{1} y_{4}-x_{4} y_{1}\right)+u_{14}\left(y_{1}-y_{4}\right)+u_{14}\left(x_{4}-x_{1}\right)\right\} \\
a_{1}=\frac{1}{2 A}\left\{u_{1}\left(x_{4} y_{14}-x_{14} y_{4}\right)+u_{1}\left(y_{4}-y_{14}\right)+u_{1}\left(x_{14}-x_{4}\right)\right\} \\
a_{2}=\frac{1}{2 A}\left\{u_{4}\left(x_{14} y_{1}-x_{1} y_{14}\right)+u_{4}\left(y_{14}-y\right)+u_{4}\left(x_{1}-x_{14}\right)\right\}
\end{array}\right\}
$$

Substituting (16) in (15)

$$
\begin{aligned}
& u=\frac{1}{2 A}\left[\left\{u_{14}\left(x_{1} y_{4}-x_{4} y_{1}\right)+u_{14}\left(y_{1}-y_{4}\right)+u_{14}\left(x_{4}-x_{1}\right)\right\}+\right. \\
& \left\{u_{1}\left(x_{4} y_{14}-x_{14} y_{4}\right)+u_{1}\left(y_{4}-y_{14}\right)+u_{1}\left(x_{14}-x_{4}\right)\right\}+ \\
& \left.\left\{u_{4}\left(x_{14} y_{1}-x_{1} y_{14}\right)+u_{4}\left(y_{14}-y_{1}\right)+u_{4}\left(x_{1}-x_{14}\right)\right\}\right]
\end{aligned}
$$

Recalling that the approximation function is

$u=N_{14} u_{14}+N_{1} u_{1}+N_{4} u_{4}$

Comparing (18) and (17) then

$$
\left.\begin{array}{l}
N_{14}=\frac{1}{2 A}\left\{\left(x_{1} y_{4}-x_{4} y_{1}\right)+\left(y_{1}-y_{4}\right) x+\left(x_{4}-x_{1}\right)\right\} y \\
N_{1}=\frac{1}{2 A}\left\{\left(x_{4} y_{14}-x_{14} y_{4}\right)+\left(y_{4}-y_{14}\right) x+\left(x_{14}-x_{4}\right)\right\} y \\
N_{4}=\frac{1}{2 A}\left\{\left(x_{14} y_{1}-x_{1} y_{14}\right)+\left(y_{14}-y_{1}\right) x+\left(x_{1}-x_{14}\right)\right\} y
\end{array}\right)
$$

But $\mathrm{A}=\frac{1}{2}\left\{\left(x_{1} y_{4}-x_{4} y_{1}\right)+\left(x_{4} y_{14}-x_{14} y_{4}\right)+\left(x_{14} y_{1}-x_{1} y_{14}\right)\right\}=168 \mathrm{~mm}^{2}$

so that

$$
\begin{aligned}
& N_{14}=\frac{1}{336}\{336+(0-16) x+(16-21)\}=\frac{1}{336}(336-16 x-5 y) \\
& N_{1}=\frac{1}{336}\{0+(16-0) x+(0-16) y\}=\frac{1}{336}(16 x-16 y) \\
& N_{4}=\frac{1}{336}\{0+(0)+(21-0) y\}=\frac{1}{336}(21 y)
\end{aligned}
$$

Substituting (22) into (18)

$$
u=\frac{1}{336}(336-16 x-5 y) u_{14}+\frac{1}{336}(16-16 y) u_{1}+\frac{1}{336}(21 y) u_{4}
$$




$$
\left.\begin{array}{l}
\frac{\partial u}{\partial x}=\frac{-16 u_{14}}{336}+\frac{16 u_{1}}{336}=\frac{-u_{14}}{21}+\frac{u_{1}}{21} \\
\frac{\partial u}{\partial y}=\frac{-5 u_{14}}{336}-\frac{16 u_{1}}{336}+\frac{21 u_{14}}{336}=\frac{-5 u_{14}}{336}-\frac{u_{1}}{21}+\frac{u_{4}}{16}
\end{array}\right)
$$

By substitutingg the first partial derivatives of the element 4 interpolarion functions in (12) with $\mathrm{dxd} y=\mathrm{A}=168 \mathrm{~mm}^{2}$

$$
x=\left(0.190 u_{14}^{2}-0.3611 u_{1} u_{14}+0.38 u_{1}^{2}-0.156 u_{4} u_{14}-0.5 u_{1} u_{4}+0.328 u_{4}^{2}\right)
$$

By differentiating w.r.t. $\mathrm{u}_{14}, \mathrm{u}_{1}$, and $\mathrm{u}_{4}$

$$
\left.\begin{array}{l}
\frac{\partial x}{\partial u_{14}}=0.418 u_{14}-0.261 u_{1}-0.156 u_{4} \\
\frac{\partial x}{\partial u_{1}}=0.76 u_{1}-0.261 u_{14}-0.5 u_{5} \\
\frac{\partial x}{\partial u_{4}}=1.656 u_{4}-0.156 u_{14}-0.5 u_{1}
\end{array}\right)
$$

\subsubsection{Element 3 interpolation and functional equation formulation}
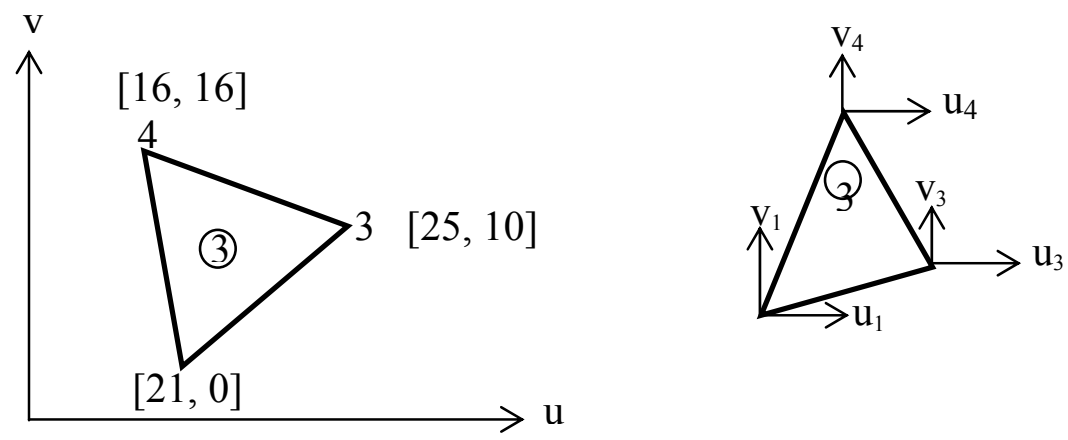

By assuming a linear approximation polynomial of the form

$$
u(x, y)=a_{0}+a_{1} x+a_{2} y
$$

and passing (26) through the nodes then

$$
\begin{aligned}
& u_{1}=a_{0}+a_{1} x_{1}+a_{2} y_{1} \\
& u_{3}=a_{0}+a_{1} x_{3}+a_{2} y_{3} \\
& u_{4}=a_{0}+a_{1} x_{4}+a_{2} y_{4}
\end{aligned}
$$


Putting the above equations in matrix form then,

$$
\left[\begin{array}{lll}
1 & x_{1} & y_{1} \\
1 & x_{3} & y_{3} \\
1 & x_{4} & y_{4}
\end{array}\right]\left[\begin{array}{l}
a_{0} \\
a_{1} \\
a_{2}
\end{array}\right]=\left[\begin{array}{ll}
u_{1} \\
u_{3} \\
u_{4}
\end{array}\right]
$$

By applying Crammers rule,

$$
\left.\begin{array}{l}
a_{0}=\frac{1}{2 A}\left\{u_{1}\left(x_{3} y_{4}-x_{4} y_{3}\right)+u_{1}\left(y_{3}-y_{4}\right)+u_{1}\left(x_{4}-x_{3}\right)\right\} \\
a_{1}=\frac{1}{2 A}\left\{u_{3}\left(x_{4} y_{1}-x_{1} y_{4}\right)+u_{3}\left(y_{4}-y_{1}\right)+u_{3}\left(x_{1}-x_{4}\right)\right\} \\
a_{2}=\frac{1}{2 A}\left\{u_{4}\left(x_{1} y_{3}-x_{3} y_{1}\right)+u_{4}\left(y_{1}-y_{3}\right)+u_{4}\left(x_{3}-x_{1}\right)\right\}
\end{array}\right\}
$$

Substituting (28) into (26)

$$
\begin{aligned}
& u=\frac{1}{2 A}\left[\left\{u_{1}\left(x_{3} y_{4}-x_{4} y_{3}\right)+u_{1}\left(y_{3}-y_{4}\right)+u_{1}\left(x_{4}-x_{3}\right)\right\}+\right. \\
& \left\{u_{3}\left(x_{4} y_{1}-x_{1} y_{4}\right)+u_{3}\left(y_{4}-y_{1}\right)+u_{3}\left(x_{1}-x_{4}\right)\right\}+ \\
& \left.\left\{u_{4}\left(x_{1} y_{3}-x_{3} y_{1}\right)+u_{1}\left(y_{1}-y_{3}\right)+u_{4}\left(x_{3}-x_{1}\right)\right\}\right]
\end{aligned}
$$

Recall that the approximation function or interpolation function is expressed as:

$u=N_{1} u_{1}+N_{3} u_{3}+N_{4} u_{4}$

Comparing (29) and (30) then,

$$
\begin{aligned}
& N_{1}=\frac{1}{2 A}\left\{\left(x_{3} y_{4}-x_{4} y_{3}\right)+\left(y_{3}-y_{4}\right) x+\left(x_{4}-x_{3}\right)\right\} y \\
& N_{3}=\frac{1}{2 A}\left\{\left(x_{4} y_{1}-x_{1} y_{4}\right)+\left(y_{4}-y_{1}\right) x+\left(x_{1}-x_{4}\right) y\right. \\
& N_{4}=\frac{1}{2 A}\left\{\left(x_{1} y_{3}-x_{3} y_{1}\right)+\left(y_{1}-y_{3}\right) x+\left(x_{3}-x_{1}\right) y\right.
\end{aligned}
$$

But $\mathrm{A}=\frac{1}{2}\left\{\left[x_{1} y_{4}-x_{4} y_{3}\right]+\left[x_{4} y_{1}-x_{1} y_{4}\right]+\left[x_{1} y_{3}-x_{3} y_{1}\right]\right\}=57 \mathrm{~mm}^{2}$

then

$$
\left.\begin{array}{l}
N_{1}=\frac{1}{114}(400-160)+(10-16) x+(16-25) y=\frac{1}{114}(240-6 x-9 y) \\
N_{3}=\frac{1}{114}(0-336)+(16-0) x+(21-16) y=\frac{1}{114}(-336+16 x-5 y) \\
N_{4}=\frac{1}{114}(525-0)+(0-10) x+(25-21) y=\frac{1}{114}(525+10 x-4 y)
\end{array}\right\}
$$


Substituting (30) into (31)

$$
\begin{aligned}
& \left.u=\frac{1}{114}(240-6 x-9 y) u_{1}+\frac{1}{114}(-336+16 x-5 y) u_{3}+\frac{1}{114}(525-10 x+4 y) u_{4}\right) \\
& \frac{\partial u}{\partial x}=\frac{-6 u_{1}}{114}+\frac{16 u_{3}}{114}-\frac{-10 u_{14}}{114} \\
& \frac{\partial \bar{u}}{\partial y}=\frac{-9 u_{1}}{114}-\frac{5 u_{3}}{114}+\frac{4 u_{4}}{114}
\end{aligned}
$$

By substitutingg the first partial derivatives of the element 4 interpolarion functions in (12) with $\mathrm{dxdy}=\mathrm{A}=57$

$$
x=0.257 u_{1}^{2}-0.224 u_{1} u_{3}+0.105 u_{1} u_{4}+0.616 u_{3}^{2}-0.789 u_{3} u_{4}+0.254 u_{4}^{2}
$$

By differentiating w.r.t. $\mathrm{u}_{1}, \mathrm{u}_{3}$, and $\mathrm{u}_{4}$

$$
\left.\begin{array}{l}
\frac{\partial x}{\partial u_{1}}=0.514 u_{1}-0.224 u_{3}-0.105 u_{4} \\
\frac{\partial x}{\partial u_{3}}=0.232 u_{3}-0.224 u_{1}-0.789 u_{4} \\
\frac{\partial x}{\partial u_{4}}=1.508 u_{4}-0.105 u_{1}-0.789 u_{3}
\end{array}\right)
$$

\subsubsection{Element 4 interpolation and functional equation formulation}

By substitutingg the first partial derivatives of the element 4 interpolarion function in (12)

$x=0.357 u_{1}^{2}-0.214 u_{1} u_{15}+0.207 u_{15}^{2}-0.5 u_{1} u_{3}-0.2 u_{3} u_{15}+0.35 u_{3}^{2}$

By differentiating w.r.t. $\mathrm{u}_{1}, \mathrm{u}_{15}$, and $\mathrm{u}_{3}$

$$
\left.\begin{array}{rl}
\frac{d x}{d u_{1}} & =0.714 u_{1}-0.2144 u_{15}-0.5 u_{3} \\
\frac{d x}{d u_{15}} & =0.414 u_{15}-0.214 u_{1}-0.2 u_{3} \\
\frac{d x}{d u_{3}} & =0.7 u_{3}-0.5 u_{1}-0.2 u_{15}
\end{array}\right)
$$




\subsubsection{Element 5 interpolation and functional equation formulation}

By substitutingg the first partial derivatives of the element 4 interpolarion functions in (12) with $\mathrm{dxdy}=\mathrm{A}=57 \mathrm{~mm}^{2}$

$x=0.35 u_{5}^{2}-0.6 u_{5} u_{9}-0.163 u_{5} u_{10}+0.4 u_{9}^{2}-0.2 u_{9} u_{10}+0.181 u_{10}^{2}$

By differentiating w.r.t. $\mathrm{u}_{5}, \mathrm{u}_{9}$, and $\mathrm{u}_{10}$

$$
\left.\begin{array}{l}
\frac{\partial x}{\partial u_{5}}=0.70 u_{5}-0.6 u_{9}-0.163 u_{10} \\
\frac{\partial x}{\partial u_{9}}=0.80 u_{9}-0.6 u_{5}-0.2 u_{10} \\
\frac{\partial x}{\partial u_{10}}=0.362 u_{10}-0.163 u_{5}-0.2 u_{9}
\end{array}\right)
$$

\subsubsection{Element 6 interpolation and functional equation formulation}

By similar procedures as above,

$x=0.254 u_{4}^{2}-0.105 u_{4} u_{5}-0.614 u_{4} u_{9}+0.616 u_{9}^{2}-0.618 u_{5} u_{9}+0.257 u_{5}^{2}$

By differentiating w.r.t. $\mathrm{u}_{4}, \mathrm{u}_{9}$, and $\mathrm{u}_{5}$

$$
\left.\begin{array}{l}
\frac{\partial x}{\partial u_{4}}=0.508 u_{5}-u_{9}-u_{10} \\
\frac{\partial x}{\partial u_{9}}=1.232 u_{9}-0.614 u_{5}-0.618 u_{5} \\
\frac{\partial x}{\partial u_{5}}=0.514 u_{5}+0.105 u_{4}-0.618 u_{9}
\end{array}\right)
$$

\subsubsection{Element 7 interpolation and functional equation formulation}

By similar procedures as above,

$$
x=0.385 u_{4}^{2}-0.469 u_{4} u_{8}-0.302 u_{4} u_{9}+0.305 u_{8}^{2}-0.141 u_{8} u_{9}+0.221 u_{9}^{2}
$$

By differentiating w.r.t. $\mathrm{u}_{4}, \mathrm{u}_{8}$, and $\mathrm{u}_{9}$ 


$$
\left.\begin{array}{c}
\frac{\partial x}{\partial u_{4}}=0.77 u_{4}-0.469 u_{8}-0.302 u_{9} \\
\frac{\partial x}{\partial u_{8}}=1.61 u_{8}-0.469 u_{4}-0.141 u_{9} \\
\frac{\partial x}{\partial u_{9}}=0.442 u_{9}-0.302 u_{4}-0.141 u_{9}
\end{array}\right)
$$

\subsubsection{Element 8 interpolation and functional equation formulation}

By similar procedures as above,

$$
x=0.215 u_{3}^{2}-0.61 u_{3} u_{8}-0.369 u_{3} u_{4}+0.295 u_{8}^{2}-0.530 u_{4} u_{8}+0.450 u_{4}^{2}
$$

By differentiating w.r.t. $\mathrm{u}_{3}, \mathrm{u}_{8}$, and $\mathrm{u}_{4}$

$$
\left.\begin{array}{rl}
\frac{\partial x}{\partial u_{3}} & =0.430 u_{3}-0.061 u_{8}-0.369 u_{4} \\
\frac{\partial x}{\partial u_{8}} & =0.590 u_{8}-0.061 u_{3}-0.530 u_{4} \\
\frac{\partial x}{\partial u_{4}} & =0.90 u_{4}-0.369 u_{3}-0.530 u_{9}
\end{array}\right)
$$

\subsubsection{Element 9 interpolation and functional equation formulation}

By similar procedures as above,

$$
x=0.5 u_{2}^{2}-0.5 u_{2} u_{3}+0.25 u_{3}^{2}+0.25 u_{15}^{2}-0.5 u_{2} u_{15}
$$

By differentiating w.r.t. $\mathrm{u}_{2}, \mathrm{u}_{3}$, and $\mathrm{u}_{15}$

$$
\left.\begin{array}{l}
\frac{\partial x}{\partial u_{2}}=u_{2}-0.5 u_{3}-0.5 u_{15} \\
\frac{\partial x}{\partial u_{3}}=0.5 u_{3}-0.5 u_{2} \\
\frac{\partial x}{\partial u_{15}}=0.5 u_{15}-0.5 u_{2}
\end{array}\right)
$$

\subsubsection{Element 10 interpolation and functional equation formulation}

By similar procedures as above, 


$$
x=0.508 u_{11}^{2}-0.61 u_{10} u_{11}+0.3 u_{10}^{2}+0.208 u_{9}^{2}-0.417 u_{9} u_{11}
$$

By differentiating w.r.t. $\mathrm{u}_{11}, \mathrm{u}_{10}$, and $\mathrm{u}_{9}$

$$
\left.\begin{array}{rl}
\frac{\partial x}{\partial u_{11}} & =1.016 u_{11}-0.6 u_{10}-0.417 u_{9} \\
\frac{\partial x}{\partial u_{10}} & =0.6 u_{10}-0.6 u_{11} \\
\frac{\partial x}{\partial u_{9}} & =0.416 u_{9}-0.417 u_{11}
\end{array}\right)
$$

\subsubsection{Element 11 interpolation and functional equation formulation}

By similar procedures as above,

$$
x=0.375 u_{13}^{2}-0.75 u_{11} u_{13}+0.542 u_{11}^{2}+0.167 u_{9}^{2}-0.333 u_{9} u_{11}
$$

By differentiating w.r.t.u $\mathrm{u}_{9}, \mathrm{u}_{13}$, and $\mathrm{u}_{1}$

$$
\left.\begin{array}{l}
\frac{\partial x}{\partial u_{9}}=0.334 u_{9}-0.333 u_{11} \\
\frac{\partial x}{\partial u_{13}}=0.75 u_{13}-0.75 u_{11} \\
\frac{\partial x}{\partial u_{11}}=1.084 u_{11}-0.75 u_{13}-0.333 u_{9}
\end{array}\right)
$$

\subsubsection{Element 12 interpolation and functional equation formulation}

By similar procedures as above,

$$
x=0.214 u_{9}^{2}+0.68 u_{9} u_{12}+0.151 u_{9} u_{13}+0.68 u_{12}^{2}-0.158 u_{12} u_{13}+0.319 u_{13}^{2}
$$

By differentiating w.r.t. $\mathrm{u}_{9}, \mathrm{u}_{12}$, and $\mathrm{u}_{13}$

$$
\left.\begin{array}{c}
\frac{\partial x}{\partial u_{9}}=0.428 u_{9}+0.684 u_{12}+0.151 u_{13} \\
\frac{\partial x}{\partial u_{12}}=1.368 u_{12}+0.684 u_{9}-0.158 u_{3} \\
\frac{\partial x}{\partial u_{13}}=0.638 u_{13}-0.151 u_{9}-0.158 u_{12}
\end{array}\right)
$$




\subsubsection{Element 13 interpolation and functional equation formulation}

By similar procedures as above,

$$
x=0.367 u_{8}^{2}-0.758 u_{8} u_{12}+0.386 u_{8} u_{9}+0.561 u_{12}^{2}-0.182 u_{9} u_{12}-0.170 u_{9}^{2}
$$

By differentiating w.r.t. $\mathrm{u}_{8}, \mathrm{u}_{12}$, and $\mathrm{u}_{9}$

$$
\left.\begin{array}{c}
\frac{\partial x}{\partial u_{8}}=0.734 u_{8}-0.758 u_{12}+0.386 u_{9} \\
\frac{\partial x}{\partial u_{12}}=1.122 u_{12}+0.758 u_{8}-0.182 u_{9} \\
\frac{\partial x}{\partial u_{9}}=0.34 u_{9}+0.386 u_{8}-0.182 u_{12}
\end{array}\right)
$$

\subsubsection{Element 14 interpolation and functional equation formulation}

By similar procedures as above,

$$
x=0.206 u_{3}^{2}-0.462 u_{3} u_{7}+0.051 u_{3} u_{8}+0.563 u_{7}^{2}-0.665 u_{7} u_{8}+0.307 u_{8}^{2}
$$

By differentiating w.r.t. $\mathrm{u}_{3}, \mathrm{u}_{7}$, and $\mathrm{u}_{8}$

$$
\left.\begin{array}{l}
\frac{\partial x}{\partial u_{3}}=0.412 u_{3}-0.462 u_{7}+0.051 u_{8} \\
\frac{\partial x}{\partial u_{7}}=1.126 u_{7}-0.462 u_{3}-0.665 u_{8} \\
\frac{\partial x}{\partial u_{8}}=0.614 u_{8}+0.51 u_{3}-0.665 u_{7}
\end{array}\right)
$$

\subsubsection{Element 15 interpolation and functional equation formulation}

By similar procedures as above,

$$
x=0.178 u_{3}^{2}+0.154 u_{3} u_{6}-0.51 u_{3} u_{7}+0.385 u_{6}^{2}-0.923 u_{6} u_{7}+0.716 u_{7}^{2}
$$

By differentiating w.r.t. $\mathrm{u}_{3}, \mathrm{u}_{6}$, and $\mathrm{u}_{7}$ 


$$
\left.\begin{array}{c}
\frac{\partial x}{\partial u_{3}}=0.356 u_{3}+0.154 u_{6}-0.51 u_{7} \\
\frac{\partial x}{\partial u_{3}}=0.356 u_{3}+0.154 u_{6}-0.51 u_{7} \\
\frac{\partial x}{\partial u_{7}}=1.432 u_{7}-0.51 u_{3}-0.923 u_{6}
\end{array}\right)
$$

\subsubsection{Element 16 interpolation and functional equation formulation}

By similar procedures as above,

$$
x=0.2 u_{3}^{2}-0.4 u_{2} u_{3}+0.513 u_{2}^{2}-0.625 u_{2} u_{6}+0.313 u_{6}^{2}
$$

By differentiating w.r.t. $\mathrm{u}_{3}, \mathrm{u}_{2}$ and $\mathrm{u}_{6}$

$$
\begin{aligned}
& \frac{\partial x}{\partial u_{3}}=0.4 u_{3}-0.4 u_{2} \\
& \frac{\partial x}{\partial u_{2}}=0.026 u_{2}-0.4 u_{3}-0.625 u_{6} \\
& \frac{\partial x}{\partial u_{6}}=0.626 u_{6}-0.625 u_{2}
\end{aligned}
$$

\section{SYSTEM ELEMENTS ASSEMBLY ALGORITHMS}

The algorithms for element assembly involves the addition of all elements contributing to minimization $\frac{d X^{e}}{d u}$, this leads to system of equations that equals the degrees of freedoms in the continuum, the derivatives are then added in a special format called assembly. There are 15 effective degrees of freedoms for the assembly of 16 elements

$\sum \frac{d X^{e}}{\partial u_{i}}=0, i=1,2,3, \ldots \ldots, 16$

For

$i=1, \sum \frac{\partial X^{e}}{\partial u_{1}}=0$

$i=2, \sum \frac{\partial X^{e}}{\partial u_{2}}=0$ 
$i=3, \Sigma \frac{\partial X^{e}}{\partial u_{3}}=0$

$i=4, \Sigma \frac{\partial X^{e}}{\partial u_{4}}=0$

$i=5, \Sigma \frac{\partial X^{e}}{\partial u_{5}}=0$

$i=6, \sum \frac{\partial X^{e}}{\partial u_{6}}=0$

$i=7, \Sigma \frac{\partial X^{e}}{\partial u_{7}}=0$

$i=8, \sum \frac{\partial X^{e}}{\partial u_{8}}=0$

$i=9, \Sigma \frac{\partial X^{e}}{\partial u_{9}}=0$

$i=10, \Sigma \frac{\partial X^{e}}{\partial u_{10}}=0$

$i=11, \Sigma \frac{\partial X^{e}}{\partial u_{11}}=0$

$i=12, \sum \frac{\partial X^{e}}{\partial u_{12}}=0$

$i=13, \sum \frac{\partial X^{e}}{\partial u_{13}}=0$

$i=14, \sum \frac{\partial X^{e}}{\partial u_{14}}=0$

$i=15, \Sigma \frac{\partial X^{e}}{\partial u_{15}}=0$

\section{ELEMENTS EQUATIONS ASSEMBLY}

All the partial derivatives resulting from the minimization scheme with respect to the fifteen (15) active degrees of freedom (DOF) are added as follows the superscripts on these equations denote element sources:

$$
\begin{aligned}
\frac{\partial X}{\partial u_{1}}=\sum \frac{\partial X^{e}}{\partial u_{1}} & =0=\frac{\partial X^{2}}{\partial u_{1}}+\frac{\partial X^{3}}{\partial u_{1}}+\frac{\partial X^{4}}{\partial u_{1}} \\
& =1.988 \mathrm{u}_{1}-0.724 \mathrm{u}_{3}-0.105 \mathrm{u}_{4}-0.5 \mathrm{u}_{5}-0.261 \mathrm{u}_{14}-0.214 \mathrm{u}_{15} \\
\frac{\partial X}{\partial u_{2}}=\Sigma \frac{\partial X^{e}}{\partial u_{2}} & =0=\frac{\partial X^{9}}{\partial u_{2}}+\frac{\partial X^{16}}{\partial u_{2}} \\
& =1.026 \mathrm{u}_{2}-0.9 \mathrm{u}_{3}-0.625 \mathrm{u}_{6}-0.5 \mathrm{u}_{15}
\end{aligned}
$$




$$
\begin{aligned}
& \frac{\partial X}{\partial u_{3}}=\sum \frac{\partial X^{e}}{\partial u_{3}}=0=\frac{\partial X^{16}}{\partial u_{3}}+\frac{\partial X^{15}}{\partial u_{3}}+\frac{\partial X^{14}}{\partial u_{3}}+\frac{\partial X^{9}}{\partial u_{3}}+\frac{\partial X^{4}}{\partial u_{3}}+\frac{\partial X^{3}}{\partial u_{3}}+\frac{\partial X^{8}}{\partial u_{3}} \\
& =-0.724 \mathrm{u}_{1}-0.9 \mathrm{u}_{2}+3.03 \mathrm{u}_{3}-1.158 \mathrm{u}_{4}+0.154 \mathrm{u}_{6}-0.972 \mathrm{u}_{7}-0.001 \mathrm{u}_{8}-0.2 \mathrm{u}_{15} \\
& \frac{\partial X}{\partial u_{4}}=\sum \frac{\partial X^{e}}{\partial u_{4}}=0=\frac{\partial X^{1}}{\partial u_{4}}+\frac{\partial X^{2}}{\partial u_{4}}+\frac{\partial X^{3}}{\partial u_{4}}+\frac{\partial X^{6}}{\partial u_{4}}+\frac{\partial X^{7}}{\partial u_{4}}+\frac{\partial X^{8}}{\partial u_{4}} \\
& =-1.158 u_{3}+5.49 u_{4}-0.395 u_{1}+0.008 u_{5}-0.469 u_{8}-1.832 u_{9}-u_{10}-0.001 u_{14} \\
& \frac{\partial X}{\partial u_{5}}=\sum \frac{\partial X^{e}}{\partial u_{5}}=0=\frac{\partial X^{1}}{\partial u_{5}}+\frac{\partial X^{5}}{\partial u_{5}}+\frac{\partial X^{6}}{\partial u_{5}} \\
& =-0.409 \mathrm{u}_{4}+1.979 \mathrm{u}_{5}-1.218 \mathrm{u}_{9}-0.163 \mathrm{u}_{10}-0.262 \mathrm{u}_{14} \\
& \frac{\partial X}{\partial u_{6}}=\sum \frac{\partial X^{e}}{\partial u_{6}}=0=\frac{\partial X^{15}}{\partial u_{6}}+\frac{\partial X^{16}}{\partial u_{6}} \\
& =1.396 \mathrm{u}_{6}-0.625 \mathrm{u}_{2}+0.154 \mathrm{u}_{3}-0.923 \mathrm{u}_{7} \\
& \frac{\partial X}{\partial u_{7}}=\sum \frac{\partial X^{e}}{\partial u_{7}}=0=\frac{\partial X^{15}}{\partial u_{7}}+\frac{\partial X^{14}}{\partial u_{7}} \\
& =2.549 u_{7}-0.972 u_{3}-0.923 u_{6}-0.665 u_{9} \\
& \frac{\partial X}{\partial u_{8}}=\sum \frac{\partial X^{e}}{\partial u_{8}}=0=\frac{\partial X^{7}}{\partial u_{8}}+\frac{\partial X^{8}}{\partial u_{8}}+\frac{\partial X^{13}}{\partial u_{8}}+\frac{\partial X^{14}}{\partial u_{8}} \\
& =0.449 \mathrm{u}_{3}-0.999 \mathrm{u}_{4}-0.665 \mathrm{u}_{7}+3.548 \mathrm{u}_{8}+0.245 \mathrm{u}_{9}-0.758 \mathrm{u}_{12} \\
& \frac{\partial X}{\partial u_{9}}=\sum \frac{\partial X^{e}}{\partial u_{9}}=0=\frac{\partial X^{13}}{\partial u_{9}}+\frac{\partial X^{12}}{\partial u_{9}}+\frac{\partial X^{11}}{\partial u_{9}}+\frac{\partial X^{10}}{\partial u_{9}}+\frac{\partial X^{7}}{\partial u_{9}}+\frac{\partial X^{6}}{\partial u_{9}}+\frac{\partial X^{5}}{\partial u_{9}} \\
& =-0.302 \mathrm{u}_{4}-1.832 \mathrm{u}_{5}+0.386 \mathrm{u}_{8}+3.851 \mathrm{u}_{9}-0.20 \mathrm{u}_{10}-0.75 \mathrm{u}_{11}+0.502 \mathrm{u}_{12}+0.151 \mathrm{u}_{13} \\
& \frac{\partial X}{\partial u_{10}}=\sum \frac{\partial X^{e}}{\partial u_{10}}=0=\frac{\partial X^{5}}{\partial u_{10}}+\frac{\partial X^{10}}{\partial u_{10}} \\
& =-0.163 \mathrm{u}_{5}-0.2 \mathrm{u}_{9}+0.962 \mathrm{u}_{10}-0.6 \mathrm{u}_{11} \\
& \frac{\partial X}{\partial u_{11}}=\sum \frac{\partial X^{e}}{\partial u_{11}}=0=\frac{\partial X^{11}}{\partial u_{11}}+\frac{\partial X^{10}}{\partial u_{11}} \\
& =-0.75 u_{9}-0.6 u_{10}+2.1 u_{11}-0.75 u_{13} \\
& \frac{\partial X}{\partial u_{12}}=\sum \frac{\partial X^{e}}{\partial u_{12}}=0=\frac{\partial X^{13}}{\partial u_{12}}+\frac{\partial X^{12}}{\partial u_{12}} \\
& =-0.158 u_{13}+0.758 u_{8}+0.502 u_{9}+2.49 u_{12}
\end{aligned}
$$




$$
\begin{aligned}
\frac{\partial X}{\partial u_{13}}=\sum \frac{\partial X^{e}}{\partial u_{13}} & =0=\frac{\partial X^{11}}{\partial u_{13}}+\frac{\partial X^{12}}{\partial u_{13}} \\
& =-0.151 u_{9}-0.75 u_{11}-0.158 u_{12}+1.388 u_{13} \\
\frac{\partial X}{\partial u_{14}}=\sum \frac{\partial X^{e}}{\partial u_{14}} & =0=\frac{\partial X^{2}}{\partial u_{14}}+\frac{\partial X^{1}}{\partial u_{14}} \\
& =-0.261 u_{1}-0.313 u_{4}-0.262 u_{5}+0.836 u_{14} \\
\frac{\partial X}{\partial u_{15}}=\sum \frac{\partial X^{e}}{\partial u_{15}} & =0=\frac{\partial X^{4}}{\partial u_{15}}+\frac{\partial X^{9}}{\partial u_{15}} \\
& =-0.214 u_{1}-0.5 u_{2}-0.2 u_{3}+0.914 u_{15}
\end{aligned}
$$

\section{APPLICATION OF BOUNDARY CONDITION}

In this work a special case where displacements at the boundaries are limited to $0.5 \mathrm{~mm}$ for an irregular continuum is considered to predict continuum displacement, strain and stress functions, while the constrained conditions are taken as zero so that by equating $\mathrm{u}_{14}=\mathrm{u}_{15}=0$ and $\mathrm{u}_{2}=\mathrm{u}_{5}=$ $\mathrm{u}_{6}=\mathrm{u}_{8}=\mathrm{u}_{10}=\mathrm{u}_{13}=0.50,(75-89)$ transform to the following:

$$
\begin{array}{ll}
1.988 u_{1}-0.724 u_{3}-0.105 u_{4} & =0.25 \\
0.900 u_{3} & =0.201 \\
-0.724 u_{1}+3.03 u_{3}-0.158 u_{4}-0.972 u_{7} & =0.374 \\
-1.158 u_{3}+5.490 u_{4}-0.395 u_{1} & =0.731 \\
-0.409 u_{4}-1.218 u_{9} & =-0.907 \\
0.154 u_{3}-0.923 u_{7} & =-0.386 \\
2.549 u_{7}-0.972 u_{3}-0.665 u_{9} & =0.462 \\
0.449 u_{3}-0.999 u_{4}-0.665 u_{7}+0.245 u_{9}-0.758 u_{12} & =-1.774 \\
3.851 u_{9}-0.302 u_{4}-0.75 u_{11}+0.502 u_{12} & =0.748 \\
-0.200 u_{9}-0.600 u_{11} & =-0.400
\end{array}
$$


$2.100 u_{11}-0.750 u_{9}$

$-0.153 u_{3}+0.502 u_{9}+2.490 u_{12}$

$-0.151 u_{9}-0.750 u_{11}-0.158 u_{12}$

$-0.261 u_{1}-0.313 u_{4}$

$-0.214 \mathrm{u}_{1}-0.200 \mathrm{u}_{3}$

$$
=0.675
$$$$
=-0.379
$$$$
=-0.694
$$$$
=0.131
$$$$
=0.250
$$

\section{SOLUTION AND POST PROCESSING FOR CONTINUUM FUNCTION}

The following nodal displacements in $\mathrm{mm}$ are further evaluated by first evaluating $\mathrm{u}_{3}=0.222$ from (91) so that other nodal values of the displacement function is as presented in Table 2 . The first partial derivatives of the interpolation function evaluated with active degree of freedom in of element with respect to the $\mathrm{x}$ axis gives the slope of the function and also gives the value of the strain as presented in Table 2. The computations are achieved with sixteen elements interpolation functions associated with the elements global coordinate axis. The strains so computed may be used with Hooke's law of elasticity to predict the stress distribution function at the respective nodes when the elastic modulus is known from literature.

Table 2: FEM Results.

\begin{tabular}{|r|c|c|}
\hline $\mathrm{n}$ (nodes) & $\mathrm{u}$ (displacement) & $\frac{\partial u}{\partial x}=\varepsilon$ (strain) \\
\hline 1 & 0.210 & 0.02 \\
\hline 2 & 0.500 & 0.01 \\
\hline 3 & 0.222 & 0.02 \\
\hline 4 & 0.059 & 0.015 \\
\hline 5 & 0.500 & 0.023 \\
\hline 6 & 0.500 & 0.015 \\
\hline 7 & 0.455 & 0.082 \\
\hline 8 & 0.500 & 0.054 \\
\hline 9 & 0.725 & 0.028 \\
\hline 10 & 0.500 & 0.01 \\
\hline 11 & 0.424 & 0.116 \\
\hline 12 & 0.500 & 0 \\
\hline 13 & 0.500 & 0.167 \\
\hline 14 & 0.000 & 0.026 \\
\hline 15 & 0.000 & 0.011 \\
\hline
\end{tabular}


The stress prediction model of a material within the elastic limit is expressed as

$\sigma=\mathrm{E} \varepsilon$

where $\mathrm{E}=$ modulus of elasticity

The excel graphics of FEM result using Table 2 of Figure 2 shows a serious indication that the minimum value of the function is between node 14 and 15 hence another extremization method is needed to point at which point of the region is this extremum.

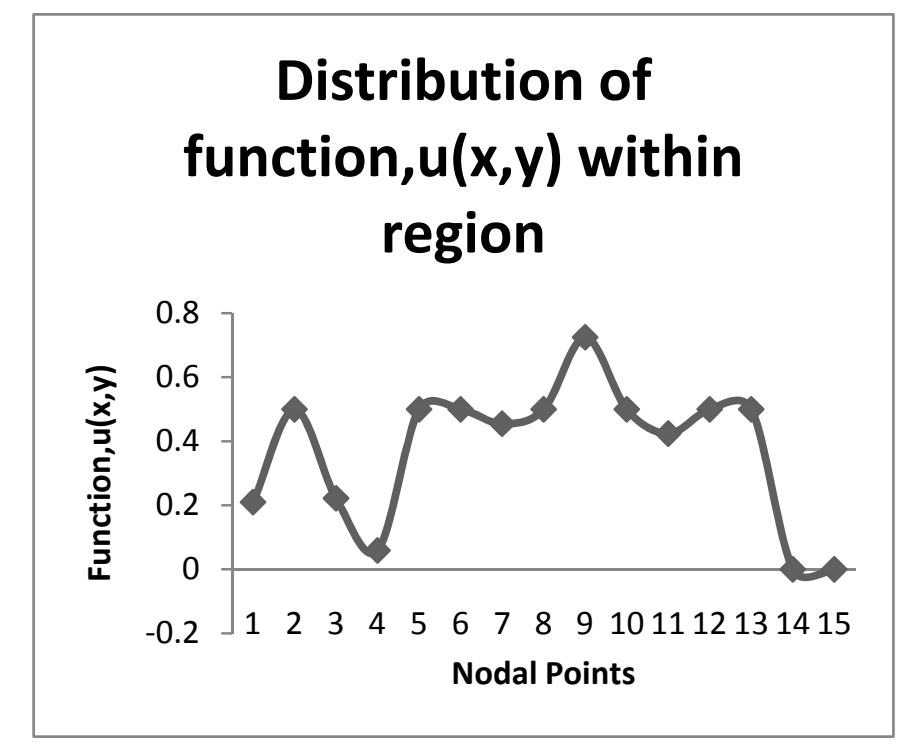

Figure 2: Distribution of Function within the Region.

\section{DISCUSSION AND VALIDATION OF RESULTS}

Regression analysis was carried out on FEM results to obtain a unified model for elements function interpolation. The regression model so obtained is further used to transform the element functional equation to aid extremization of FEM results.

\subsection{Regression Analysis}

Multiple linear regression analysis was carried out on finite element results to obtain the following model for the region. By employing the classical multiple linear regression equation of the form

$\mathrm{u}(\mathrm{x}, \mathrm{y})=\mathrm{a}_{\mathrm{o}}+\mathrm{a}_{1} \mathrm{x}+\mathrm{a}_{2} \mathrm{y}$ 
a regression model for the FEM is obtained with Table 3 and expressed as (107).

$u(x, y)=0.065+0.0036 x+0.0130 y$

The goodness of fit of regression was evaluated to obtain: Coefficient of determination, $\mathrm{r}^{2}=0.52$, correlation coefficient, $\mathrm{r}=0.72$, standard error, $\mathrm{s}_{\mathrm{e}}=0.1$

where $\mathrm{u}=$ field function evaluated through FEM

$\mathrm{u}^{1}=$ average of FEM function

up $=$ field function predicted with regression model

Table 2 and Figure 2 show the variation of the function within the region. Continuum fluid elements in heat and mass transfer operations associated with pipeline transportation can elegantly be analyzed following the procedure of this work. The FEM developed can be applied in the evaluation of the stress distribution in irregular shaped continuum whose boundary conditions are specified such as in the evaluation of displacement in structures and solid mechanics problems, evaluation of temperature distribution in heat conduction problems, evaluation of displacement potential in acoustic fluids, evaluation of pressure in potential flows ,evaluation of velocity in general flows, evaluation of electric potential in electrostatics and in evaluation of magnetic potential in magnetostatics.

\subsection{Extremization of Functional: Extremization by Lagrange Multipliers Approach}

In order to further analyse the FEM results, the functional, $\chi$ of any element is transformed to a function of $(\mathrm{x}, \mathrm{y})$ using the regression model of (107) to obtain:

$$
\begin{aligned}
x=f(x, y)= & 0.000042 x+0.0017 y+0.000059 x^{2}+0.00034 y^{2}+0.00015 x y+0.00847 \\
& =(108)
\end{aligned}
$$

Figure 4a,b and c show versions of 3D plots of function using Matlab for (108)

The objective function

$f(x, y)=0.000042 x+0.0017 y+0.000059 x^{2}+0.00034 y^{2}+0.00015 x y+0.00847$

subject to the constraint relations

$\mathrm{u}(\mathrm{x}, \mathrm{y})=0.5+0.0225 \mathrm{x} \quad=0.5$

$\mathrm{u}(\mathrm{x}, \mathrm{y})=-0.0201 \mathrm{x}+0.0238 \mathrm{y}=0$

derived for nodes 14 and 10 of elements 1 and 5 at the boundaries. 
Table 3 Computations For Regression and Error Analysis of FEM Results.

\begin{tabular}{|c|c|c|c|c|c|c|c|c|c|c|c|}
\hline $\mathrm{N}$ & $\mathrm{x}$ & $\mathrm{y}$ & $\mathrm{u}$ & $x^{2}$ & $y^{2}$ & $\mathrm{x} * \mathrm{y}$ & $\mathrm{x} * \mathrm{u}$ & $\mathrm{y} * \mathrm{u}$ & up & $\left(u-u^{1}\right)^{2}$ & $(\mathrm{u}-\mathrm{up})^{2}$ \\
\hline 1 & 21 & 0.0 & 0.2100 & 441 & 0.0000 & 0.0000 & 4.4100 & 0.0000 & 0.1406 & 0.0266 & 0.004816 \\
\hline 2 & 35 & 10 & 0.5000 & 1225 & 100 & 350 & 17.5000 & 5.0000 & 0.321 & 0.0161 & 0.032041 \\
\hline 3 & 25 & 10 & 0.2220 & 625 & 100 & 250 & 5.5500 & 2.2200 & 0.285 & 0.0228 & 0.003969 \\
\hline 4 & 16 & 16 & 0.0590 & 256 & 256 & 256 & 0.944 & 0.9440 & 0.3306 & 0.0986 & 0.073767 \\
\hline 5 & 0.0 & 21 & 0.5000 & 0.0000 & 441 & 0.0000 & 0.0000 & 10.5000 & 0.338 & 0.0161 & 0.026244 \\
\hline 6 & 35 & 18 & 0.5000 & 1225 & 324 & 630 & 17.5000 & 9.0000 & 0.425 & 0.0161 & 0.005625 \\
\hline 7 & 29 & 19 & 0.4550 & 841 & 361 & 551 & 13.195 & 8.6450 & 0.4164 & 0.0067 & 0.00149 \\
\hline 8 & 22 & 23 & 0.5000 & 484 & 529 & 506 & 11.000 & 11.5000 & 0.4432 & 0.0161 & 0.003226 \\
\hline 9 & 10 & 25 & 0.7250 & 100 & 625 & 250 & 7.2500 & 18.1250 & 0.426 & 0.1239 & 0.089401 \\
\hline 10 & 0.0 & 37 & 0.5000 & 0.0000 & 1369 & 0.0000 & 0.0000 & 18.5000 & 0.546 & 0.0161 & 0.002116 \\
\hline 11 & 10 & 37 & 0.424 & 100 & 1369 & 370 & 4.2400 & 15.6880 & 0.582 & 0.0026 & 0.024964 \\
\hline 12 & 19 & 29 & 0.5000 & 361 & 841 & 551 & 9.5000 & 14.5000 & 0.5104 & 0.0161 & 0.000108 \\
\hline 13 & 18 & 37 & 0.5000 & 324 & 1369 & 666 & 9.0000 & 18.5000 & 0.6108 & 0.0161 & 0.012277 \\
\hline 14 & 0.0 & 0.0 & 0.0000 & 0.0000 & 0.0000 & 0.0000 & 0.0000 & 0.0000 & 0.065 & 0.1391 & 0.004225 \\
\hline 15 & 35 & 0.0 & 0.0000 & 1225 & 0.0000 & 0.0000 & 0.0000 & 0.0000 & 0.191 & 0.1391 & 0.036481 \\
\hline sum & 275 & 282 & 5.595 & 7207 & 7684 & 4380 & 100.089 & 133.122 & 5.631 & 0.6721 & 0.32075 \\
\hline $\mathrm{N}$ & $\mathrm{x}$ & $\mathrm{y}$ & $\mathrm{u}$ & $x^{2}$ & $y^{2}$ & $\mathrm{x} * \mathrm{y}$ & $\mathrm{x} * \mathrm{u}$ & $\mathrm{y} * \mathrm{u}$ & up & $\left(u-u^{1}\right)^{2}$ & $(\mathrm{u}-\mathrm{up})^{2}$ \\
\hline 1 & 21 & 0.0 & 0.2100 & 441 & 0.0000 & 0.0000 & 4.4100 & 0.0000 & 0.1406 & 0.0266 & 0.004816 \\
\hline 2 & 35 & 10 & 0.5000 & 1225 & 100 & 350 & 17.5000 & 5.0000 & 0.321 & 0.0161 & 0.032041 \\
\hline 3 & 25 & 10 & 0.2220 & 625 & 100 & 250 & 5.5500 & 2.2200 & 0.285 & 0.0228 & 0.003969 \\
\hline 4 & 16 & 16 & 0.0590 & 256 & 256 & 256 & 0.944 & 0.9440 & 0.3306 & 0.0986 & 0.073767 \\
\hline 5 & 0.0 & 21 & 0.5000 & 0.0000 & 441 & 0.0000 & 0.0000 & 10.5000 & 0.338 & 0.0161 & 0.026244 \\
\hline 6 & 35 & 18 & 0.5000 & 1225 & 324 & 630 & 17.5000 & 9.0000 & 0.425 & 0.0161 & 0.005625 \\
\hline 7 & 29 & 19 & 0.4550 & 841 & 361 & 551 & 13.195 & 8.6450 & 0.4164 & 0.0067 & 0.00149 \\
\hline 8 & 22 & 23 & 0.5000 & 484 & 529 & 506 & 11.000 & 11.5000 & 0.4432 & 0.0161 & 0.003226 \\
\hline 9 & 10 & 25 & 0.7250 & 100 & 625 & 250 & 7.2500 & 18.1250 & 0.426 & 0.1239 & 0.089401 \\
\hline 10 & 0.0 & 37 & 0.5000 & 0.0000 & 1369 & 0.0000 & 0.0000 & 18.5000 & 0.546 & 0.0161 & 0.002116 \\
\hline 11 & 10 & 37 & 0.424 & 100 & 1369 & 370 & 4.2400 & 15.6880 & 0.582 & 0.0026 & 0.024964 \\
\hline 12 & 19 & 29 & 0.5000 & 361 & 841 & 551 & 9.5000 & 14.5000 & 0.5104 & 0.0161 & 0.000108 \\
\hline 13 & 18 & 37 & 0.5000 & 324 & 1369 & 666 & 9.0000 & 18.5000 & 0.6108 & 0.0161 & 0.012277 \\
\hline 14 & 0.0 & 0.0 & 0.0000 & 0.0000 & 0.0000 & 0.0000 & 0.0000 & 0.0000 & 0.065 & 0.1391 & 0.004225 \\
\hline 15 & 35 & 0.0 & 0.0000 & 1225 & 0.0000 & 0.0000 & 0.0000 & 0.0000 & 0.191 & 0.1391 & 0.036481 \\
\hline sum & 275 & 282 & 5.595 & 7207 & 7684 & 4380 & 100.089 & 133.122 & 5.631 & 0.6721 & 0.32075 \\
\hline
\end{tabular}


By taking partial derivatives of Lagrange expression

$$
\begin{aligned}
\mathrm{L}\left(\mathrm{x}, \mathrm{y}, \lambda_{1}, \lambda_{2}\right)=\mathrm{f}(\mathrm{x}, \mathrm{y})+ & \lambda_{1} \mathrm{~g}_{1}(\mathrm{x}, \mathrm{y})+\lambda_{2} \mathrm{~g}_{2}(\mathrm{x}, \mathrm{y}) \\
=0.000042 \mathrm{x} & +0.0017 \mathrm{y}+0.000059 \mathrm{x}^{2}+0.00034 \mathrm{y}^{2}+0.00015 \mathrm{xy}+0.00847 \\
& +\lambda_{1}(0.0225 \mathrm{x})+\lambda_{2}(-0.0201 \mathrm{x}+0.0238 \mathrm{y})
\end{aligned}
$$

to obtain the following relations

$$
\begin{aligned}
& \frac{\partial \mathrm{L}}{\partial \mathrm{x}}=0.000042+0.0001 \mathrm{x}+0.00015 \mathrm{y}+0.0225 \lambda_{1}-0.0201 \lambda_{2}=0 \\
& \frac{\partial \mathrm{L}}{\partial \mathrm{y}}=0.0017+0.0068 \mathrm{y}+0.00015 \mathrm{x}+0.0225 \lambda_{1}+0.0238 \lambda_{2}=0 \\
& \frac{\partial \mathrm{L}}{\partial \lambda_{1}}=0.0225 \mathrm{x} \quad=0 \\
& \frac{\partial \mathrm{L}}{\partial \lambda_{2}}=0.0201 \mathrm{x}+0.0238 \mathrm{y} \quad=0
\end{aligned}
$$

By solving (107)- (110) from(109)

$x=y=0, \lambda_{1}=-0.0356, \lambda_{2}=-0.0378$

By substituting the variables in (108) the optimum value of the function is obtained as

$$
\begin{aligned}
\mathrm{u}(\mathrm{x}, \mathrm{y})=\mathrm{f}(\mathrm{x}, \mathrm{y})=0.00847 & \\
\mathrm{x}=\mathrm{f}(\mathrm{x}, \mathrm{y})= & 0.000042 \mathrm{x}+0.0017 \mathrm{y}+0.000059 \mathrm{x}^{2}+0.00034 \mathrm{y}^{2}+0.00015 \mathrm{xy} \\
& +0.00847 \quad \text { (108) }
\end{aligned}
$$

The prediction of functional, $\chi$ with (108) are presented in Table 4 using excel package to draw conclusion with the FEM and multiple linear regression results of Table 3. 
Table 4: Prediction of Functional with Equation (108).

\begin{tabular}{|l|l|l|r|}
\hline $\mathrm{N}$ & $\mathrm{X}$ & $\mathrm{Y}$ & $\mathrm{X}$ \\
\hline 1 & 21 & 0 & 0.035371 \\
\hline 2 & 35 & 10 & 0.185715 \\
\hline 3 & 25 & 10 & 0.134895 \\
\hline 4 & 16 & 16 & 0.176886 \\
\hline 5 & 0 & 21 & 0.19411 \\
\hline 6 & 35 & 18 & 0.317475 \\
\hline 7 & 29 & 19 & 0.296997 \\
\hline 8 & 22 & 23 & 0.33281 \\
\hline 9 & 10 & 25 & 0.30729 \\
\hline 10 & 0 & 37 & 0.53683 \\
\hline 11 & 10 & 37 & 0.59865 \\
\hline 12 & 19 & 29 & 0.448457 \\
\hline 13 & 18 & 37 & 0.656602 \\
\hline 14 & 0 & 0 & 0.00847 \\
\hline 15 & 35 & 0 & 0.082215 \\
\hline
\end{tabular}

Tables 3 and 4 are compared for $\mathrm{u}$, up and their functional, $\chi$ are found approximate.

\subsubsection{Extremization by Lagrange gradient search approach}

The extremum conditions for continuous and differentiable functions are defined [1] as follows:

$$
\begin{aligned}
& f_{x}=0.000042+0.0001 x+0.00015 y=0 \\
& f_{y}=0.0017+0.000068 y+0.00015 x=0 \\
& f_{x x}=0.0001 \\
& f_{y y}=0.00068
\end{aligned}
$$

Since $\mathrm{f}_{\mathrm{xx}}$ and $\mathrm{f}_{\mathrm{yy}}>0 \Rightarrow$ minimum extremum or local extremum exists.

The extremum at the interior points $\left(\mathrm{x}_{0}, \mathrm{y}_{0}\right)$ is evaluated by solving simultaneous equation formed by (100) and (101) to obtain $\mathrm{x}=4.9767, \mathrm{y}=-3.5978$. By substituting this value in equation (108) the function is obtained as 0.006 , representing the extrema (minimum) value of the function $\mathrm{u}(\mathrm{x}, \mathrm{y})$ within the region. 


\subsubsection{Extremization by Lagrange multipliers approach}

By expressing (108) in the form

$f(x, y)=0.000042 x+0.0017 y+0.000059 x^{2}+0.00034 y^{2}+0.00015 x y+0.00847$

Subject to the constraint relations

$\mathrm{u}(\mathrm{x}, \mathrm{y})=0.5+0.0225 \mathrm{x} \quad=0.5$

$\mathrm{u}(\mathrm{x}, \mathrm{y})=-0.0201 \mathrm{x}+0.0238 \mathrm{y}=0$

derived for nodes 14 and 10 of elements 1 and 5 at the boundaries.

By taking partial derivatives of Lagrange expression

$$
\begin{aligned}
\mathrm{L}\left(\mathrm{x}, \mathrm{y}, \lambda_{1}, \lambda_{2}\right)= & (\mathrm{x}, \mathrm{y})+\lambda_{1} \mathrm{~g}_{1}(\mathrm{x}, \mathrm{y})+\lambda_{2} \mathrm{~g}_{2}(\mathrm{x}, \mathrm{y}) \\
=0.000042 \mathrm{x}+0.0017 \mathrm{y}+0.000059 \mathrm{x}^{2}+0.00034 \mathrm{y}^{2}+0.00015 \mathrm{xy}+0.00847 & +\lambda_{1}(0.0225 \mathrm{x})+\lambda_{2}(-0.0201 \mathrm{x}+0.0238 \mathrm{y})
\end{aligned}
$$

to obtain the following relations

$$
\begin{aligned}
& \frac{\partial \mathrm{L}}{\partial \mathrm{x}}=0.000042+0.0001 \mathrm{x}+0.00015 \mathrm{y}+0.0225 \lambda_{1}-0.0201 \lambda_{2}=0 \\
& \frac{\partial \mathrm{L}}{\partial \mathrm{y}}=0.0017+0.0068 \mathrm{y}+0.00015 \mathrm{x}+0.0225 \lambda_{1}+0.0238 \lambda_{2}=0 \\
& \frac{\partial \mathrm{L}}{\partial \lambda_{1}}=0.0225 \mathrm{x} \quad=0 \\
& \frac{\partial \mathrm{L}}{\partial \lambda_{2}}=0.0201 \mathrm{x}+0.0238 \mathrm{y} \quad=0
\end{aligned}
$$

By solving (123)- (126) starting from(125)

$x=y=0, \lambda_{1}=-0.0356, \lambda_{2}=-0.0378$

By substituting the variables in (108) the optimum value of the function is obtained as $\mathrm{u}(\mathrm{x}, \mathrm{y})=\mathrm{f}(\mathrm{x}, \mathrm{y})=0.00847$. This value compares favourably with the prediction of 0.006 of gradient search method showing agreement with the graphics of Figure 2 and Figure 3. 
a)

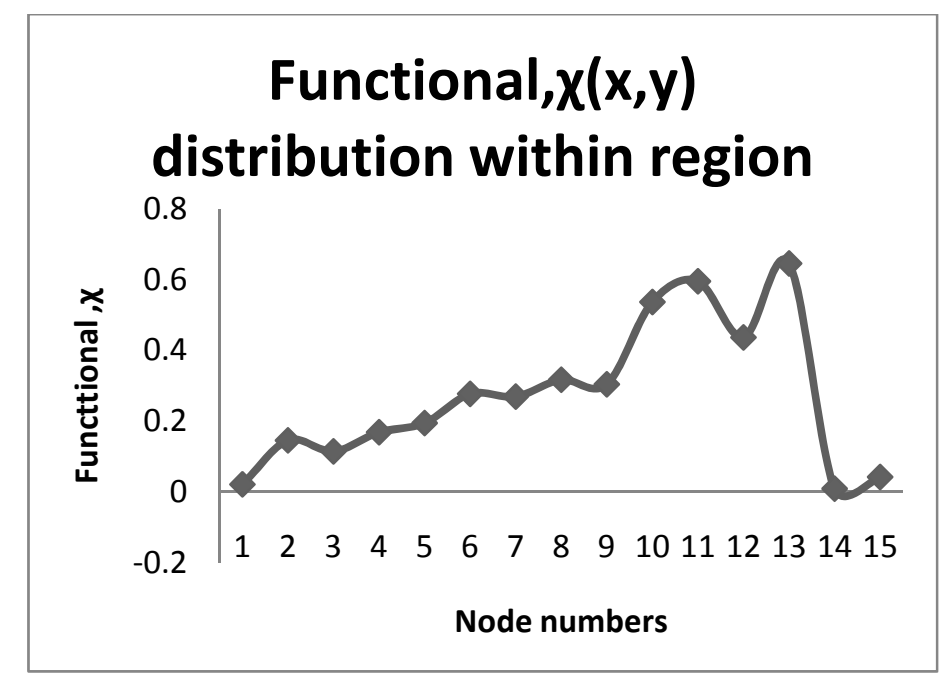

b)

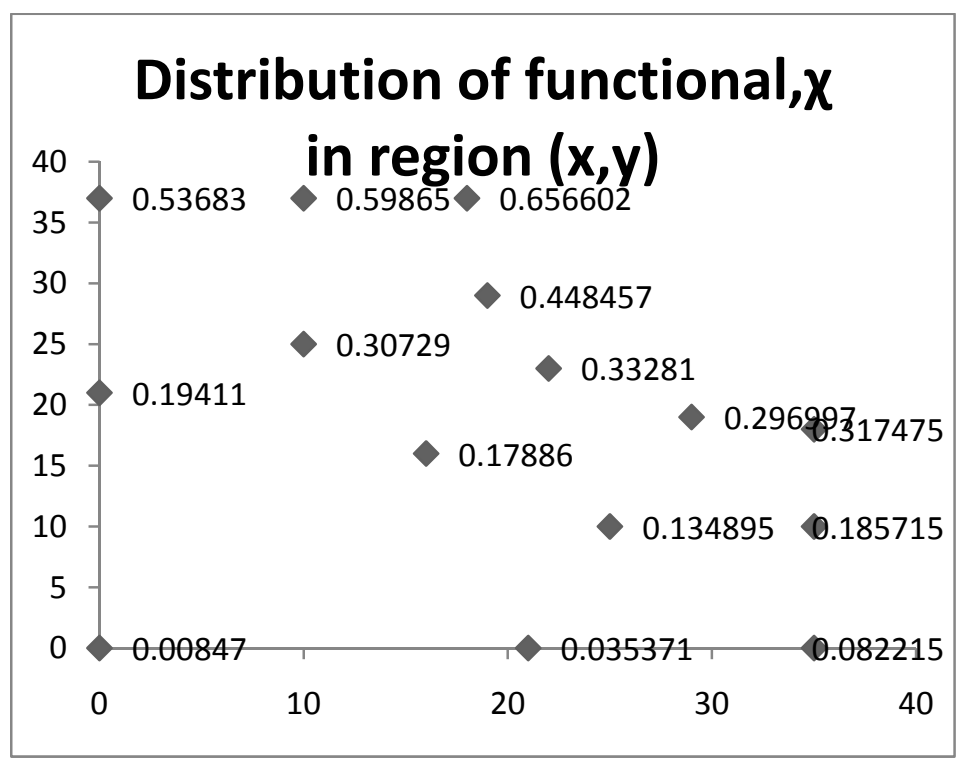

Figure 3a, b Distribution of function within the Region. 

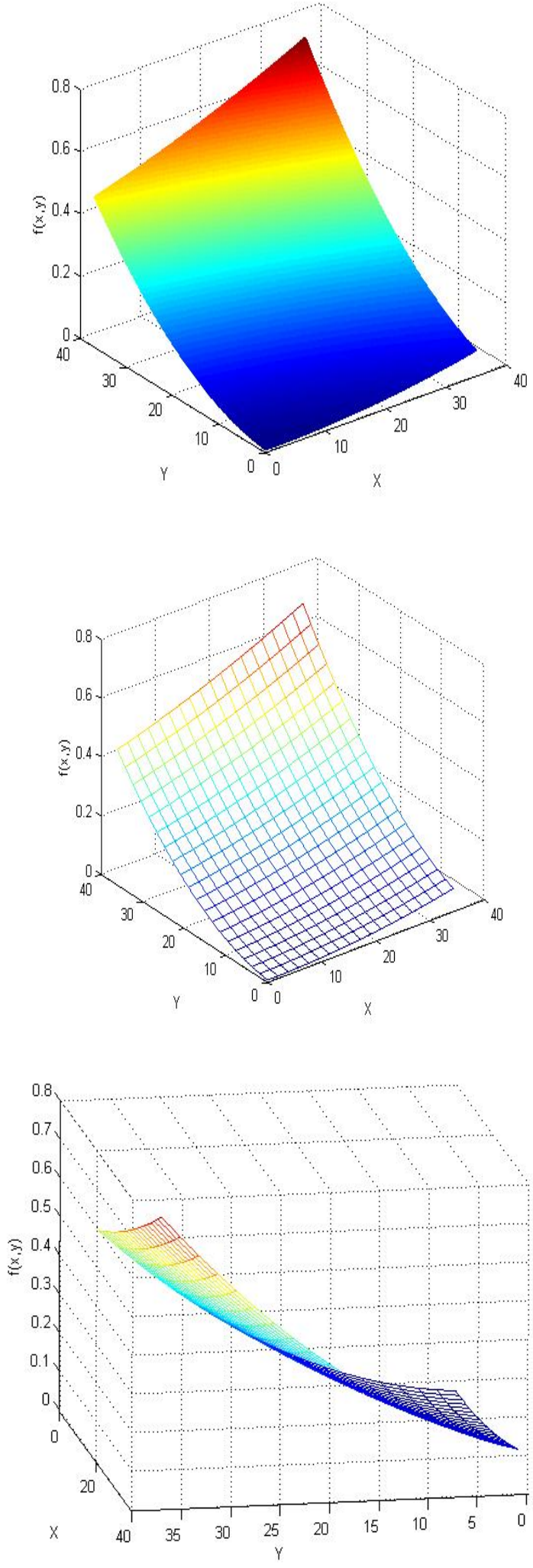

Figure $4 \mathrm{a}, \mathrm{b}$ and $\mathrm{c}$ Versions of 3D Surface Plots of Function. 


\section{CONCLUSSIONS}

The methods of this article apply to:

1. Solution of boundary value engineering phenomena whose function can be expressed as partial differential equation.

2. Solution of of displacement in structures and solid mechanics problems, temperature distribution in heat conduction problems, displacement potential in acoustic fluids, pressure in potential flows, velocity in general flows, electric potential in electrostatics magnetic potential in magnetostatics, torsion of non - homogenous shaft, flow through an anisotropic porous foundation, axi - symmetric heat flow, hydrodynamic pressures on moving surfaces

3. Solution of time dependent field problems such as creep, fracture and fatigue.

4. Equations (97) and (98) are recommended for the prediction of possible values of the displacement function of GRP composites region from where other properties of the region could be evaluated.

5. A unified computational model with standard error of 0.15 and correlation coefficient of 0.72 was developed to aid analysis and easy prediction of regional function with which the continuum function was successfully modeled and optimized through gradient search and Lagrange multipliers approach.

6. The MatLab 3-D graphics of Figure 4 show potential trend of function within the regionwith minimum and maximum at the boundaries.

\section{REFERENCES}

[1] Amazigo, J.C and Rubenfield, L.A (1980). Advanced Calculus and its application to the Engineering and Physical Sciences, John Wiley and sons Publishing, New York, pp.130

[2] Zienkiewicz,O.C., and Cheung, Y.K,(1967). The Finite Element in Structural and Continuum Mechanics, McGraw-Hill Publishing Coy Ltd, London, pp.148

[3] Sundaram,V.,Balasubramanian,R.,Lakshminarayanan,K.A.,(2003) Engineering Mathematics, Vol.3,VIKAS Publishing House LTD, New Delhi,pp.173.

[4] Ihueze, C. C,Umenwaliri,S.Nand Dara,J.E (2009) Finite Element Approach to Solution of Multidimensional Field Functions, African Research Review: An International MultiDisciplinary Journal, Vol.3 (5), October, 2009, pp.437-457.

[5] Astley, R.J., (1992), Finite Elements in Solids and Structures, Chapman and Hall Publishers, UK, pp.77

[6] Ihueze, C. C. (2010). The Galerki Approach for Finite Elements of Field Functions: The Case of Buckling in GRP. Journal of Minerals and Materials Characterization and Engineering (JMMCE), Vol.9, No.4, pp.389-409 
[7] Ihueze, Chukwutoo. C., (2010). Finite Elements in the Solution of Continuum Field Problems, Journal of Minerals and Materials Characterization and Engineering (JMMCE), Vol.9, No.5.pp.427-452

[8] Canale, R.P and Chapra, S.C (1998), Numerical Methods for Engineers, McGraw-Hill Publishers, $3^{\text {rd }}$ edition, Boston, N.Y,pp.849-853 